\title{
RECONSTRUIR LA IDENTIDAD SOCIAL DE LAS MUJERES PARA LA TRANSFORMACIÓN PACÍFICA DE LOS CONFLICTOS
}

SONIA PARÍS ALBERT

Universitat Jaume I, Castellón

\section{INTRODUCCIÓN}

Este trabajo quiere enfatizar las aportaciones que las mujeres pueden hacer a la transformación pacífica de los conflictos. Por esta razón, se recordará en qué medida los procesos de regulación pacífica pueden verse favorecidos con la colaboración activa de las mujeres. Con el fin de lograr este objetivo, en primer lugar, se revisará la influencia que ha tenido la apariencia física del cuerpo en la forma en la que, tradicionalmente, hemos percibido socialmente a las mujeres. Este recorrido nos permitirá deconstruir, en segundo lugar, esta imagen, y reconstruir, en último lugar, su identidad de una manera más activa y comprometida con las problemáticas actuales $y$, principalmente, con la transformación pacífica de los conflictos. Es decir, la transformación positiva de las situaciones conflictivas se convertirá en uno de los marcos de acción de las mujeres, que nos ayudará a la reconstrucción de su identidad, tal y como en estas páginas se propone. Sin embargo, no se quiere afirmar que la metodología de la transformación pacífica de los conflictos depende, exclusivamente, de las aportaciones de las mujeres, sino que su práctica puede verse beneficiada si se tienen en cuenta tanto los saberes propios de las mujeres como los de los hombres.

\section{LA RELACIÓN ENTRE EL CUERPO Y LA IDENTIDAD DE LA MUJER}

Una cuestión que podríamos plantearnos es si el hecho de nacer con un cuerpo de mujer es un marcador que determina las funciones sociales a realizar por quien posee este cuerpo de mujer y, en este sentido, la identidad y esencia femenina en nuestra vida. Esta reflexión aparece de igual forma en un artículo 
de Pintos Peñaranda ${ }^{1}$ en el que se pregunta lo siguiente: "interviene nuestro cuerpo de forma importante en el hacernos humanos en un mundo de sentido humano?(...) El proceso por el que cada criatura nacida de mujer se hace humana al integrarse en una cultura, ¿es un proceso que se lleva a cabo dependiendo de que su cuerpo traiga consigo los atributos de macho o que los traiga de hembra?».

A partir de una perspectiva fenomenológica se puede entender que es nuestro cuerpo el que favorece el reconocimiento de la mujer como mujer, y el que, por lo tanto, limita sus funciones económicas, sociales y políticas a las que han venido siendo construidas, tradicionalmente. En este caso, la Fenomenología afirma que "yo soy mi cuerpo", y debido a que es por medio de mi cuerpo que vivo en un mundo humano, mi cuerpo es, a un mismo tiempo, percepción y comportamiento ${ }^{2}$.

Mi cuerpo es percepción porque mediante su función sensorial, visual, acústica y táctil percibo las cosas que me rodean como ellas son, y me comunico con las personas que me envuelven. En este sentido, mi cuerpo me posibilita aprender cómo se responde a los conflictos en el contexto social en el que me encuentro; cuáles son los roles de género que existen; qué funciones tiene la mujer; y cuál es la esencia de lo femenino. Se produce así un aprendizaje intersubjetivo que me lleva a utilizar las mismas pautas de comportamiento que las de las personas con las que convivo; a responder a los conflictos con los mismos patrones de conducta; y a reconocer la identidad de las mujeres con los mismos criterios. Por lo tanto, cada sujeto es siempre un reflejo de lo social desde tres niveles: el nivel del pensamiento, de los sentimientos y de la conducta. Cada mujer piensa, siente y actúa según las formas en las que lo hacen quienes la rodean y con quienes, contínuamente, se interrelaciona. Por esta razón, decía Husserl ${ }^{3}$ que "la realidad cuerpo corporal expresa una regulación intersubjetiva», o Sartre ${ }^{4}$ señalaba que "soy algo que no he elegido ser»; y que «el cuerpo-para-el-otro es el cuerpo-para-nosotros».

Mi cuerpo es comportamiento porque sus movimientos sirven de modelo a quien se encuentra delante de mí, y es a través del aprendizaje intersubjetivo que "los otros y otras» reproducen mis mismos movimientos, al igual que yo ya también he hecho con anterioridad, y en relación con los movimientos de las otras personas.

"Mi cuerpo, y el cuerpo del otro, son ambos portadores de comportamiento. La intersubjetividad se me da primeramente bajo la forma de una intercorporalidad y que, en mi vida empírica, lo que asumo de mi cultura me ha venido o me viene co-

1. Pintos Peñaranda, $M^{a}$ Luz: "Cuerpo de mujer y violencia simbólica: una realidad universal», en Jacinto Rivera de Rosales y Ma del Carmen López Sáenz (coords.): El cuerpo. Perspectivas Filosóficas, Madrid, Universidad Nacional de Educación a Distancia, 2003, pp. 291 y 292.

2. MerleaU-PONTY, Maurice: Fenomenología de la percepción, Barcelona, Península, 1975.

3. HUSSERL, Edmund: Ideas relativas a una fenomenología pura y una filosofía fenomenológica, II, México, UNAM, 1997, p. 329.

4. SARTRE, Jean-Paul: El ser y la nada, Madrid, Alianza Universidad, 1989, p. 380. 
Reconstruir la identidad social de las mujeres para la transformación pacífica de los...

municado por los actos corporales de los otros [...] y, una vez que asumo en mi vida la social, en adelante también lo habré de expresar simbólicamente ante los otros por medio de mi cuerpo» ${ }^{5}$.

Más aún, la apariencia física de mi cuerpo es la que determina mi identidad, mi esencia como humana y mi papel en la sociedad. Así, el sólo hecho de ver un cuerpo de mujer lleva a interpretar que quien lo posee debería quedar relegada a la vida privada, al mismo tiempo, que es ese cuerpo de mujer el que promueve la concepción de la mujer como un ser subordinado, y el que "automáticamente provoca en su entorno inmediato el inicio sobre él de las estrategias de dominación» ${ }^{6}$. En este sentido, puede relacionarse con las propuestas de Reardon ${ }^{7}$ cuando sugiere que es el miedo a lo diferente una de las principales causas de la violencia de género. De este modo, podría interpretarse que el miedo frente a lo diferente de nuestro cuerpo de mujer provoca la respuesta inmediata de la violencia, así como el ejercicio del poder autoritario del hombre sobre la mujer. En cambio, desde la Filosofía para la Paz de la Cátedra UNESCO de Filosofía para la Paz de la Universitat Jaume I de Castellón advertimos la necesidad de regular este miedo con alternativas pacíficas basadas en el reconocimiento y en la comunicación.

Tal es la influencia del cuerpo femenino que se ha llegado a resaltar la sexualidad y la reproducción como dos de los rasgos más característicos de la identidad de la mujer. Este hecho ha puesto el énfasis en el reconocimiento de la mujer como un objeto de intercambio que produce un placer sexual y que es fuente de fecundidad en la familia. Así, la mujer no ha sido dueña de su cuerpo, sexualidad y reproducción, sino que se le ha usurpado todo ello, y se le ha entregado su control al hombre. Por esta razón, desde el determinismo biológico, se ha entendido que la mujer es útero, y como tal, "tiene menstruaciones y está dominada por ellas, es o no es virgen, puede quedar embarazada y puede parir. La menstruación, la virginidad, el embarazo y el parto, a esto queda reducida la esencia de la mujer en su sentido primordial» ${ }^{8}$. En este sentido, sus actividades han quedado relegadas al ámbito de lo privado, a la vida familiar, al cuidado de los hijos y las personas mayores, y se ha destacado su capacidad para el desarrollo de la ternura, el respeto, las caricias y el amor. Estos rasgos la han aproximado a la Cultura de la Paz y la han contrapuesto a los hombres, quienes han sido identificados, tradicionalmente, como más cercanos a la cultura de la guerra. De este modo, se ha construido una dicotomía entre la mujer y el hombre que ha causado la separación entre el ámbito de lo privado y de lo público, y que ha impedido aprovechar los saberes de las mujeres para la construcción de una convivencia armónica y en paz.

5. intos Peñaranda, Ma Luz: Op. cit, p. 297.

6. Ibíd, p. 299.

7. REARDON, Betty: Sexism and the war system, New York, Teachers College, Columbia University, 1985.

8. Pintos Peñaranda, Ma Luz: Op. cit, p. 307. 


\begin{tabular}{|c|c|}
\hline \multicolumn{2}{|c|}{ Dicotomía Mujer y Hombre } \\
\hline Mujer & Hombre \\
\hline Privado & Público \\
Cultura de la Paz & Cultura de la Guerra \\
Ternura & Violencia \\
Solidaridad & Valentía \\
Respeto & Esfuerzo \\
Amor & Odio, rencor e ira \\
\hline
\end{tabular}

Nuestro cuerpo ha participado en la configuración de nuestra identidad como mujeres, y mediante este cuerpo, hemos aprendido, también, qué es ser mujer y cómo se es mujer. Durante generaciones hemos transmitido estas mismas pautas de conducta, y aunque en la actualidad las cosas han adquirido un nuevo color, todavía hay muchas acciones que podemos realizar. En este sentido, en la siguientes páginas deconstruiré la dicotomía entre mujer y hombre con el fin de reconstruir una nueva forma de reconocer la identidad de la mujer. Ello lo realizaré teniendo en cuenta las aportaciones que las mujeres pueden hacer a la transformación pacífica de los conflictos como uno de sus posibles campos de acción.

Mi objetivo es mostrar que no por el simple hecho de poseer un cuerpo de mujer nuestras funciones han de ser limitadas al ámbito privado, sino que, también, siendo mujeres podemos hacer uso de nuestros saberes y conocimientos para la construcción de la paz, a raíz de una participación activa y comprometida con los procesos de paz, y con la transformación del sufrimiento humano por medios pacíficos. De esta forma, tendrá lugar un aprendizaje intersubjetivo que nos permitirá reconocer otra forma de ser mujer, y que enfatizará su papel activo, participativo y comprometido con las distintas maneras que tenemos de podernos hacer las paces.

\section{LA MUJER EN LA TRANSFORMACIÓN PACÍFICA DE LOS CONFLICTOS}

Si en el punto anterior se ha señalado que, tradicionalmente, las funciones de las mujeres han sido limitadas al ámbito privado, así como la influencia de la apariencia física de su cuerpo sobre ello, en este apartado me propongo deconstruir la dicotomía entre mujer y hombre que, en gran medida, ha sido la causa de esta percepción. En este caso y siguiendo las investigaciones de la Cátedra UNESCO de Filosofía para la Paz de la Universitat Jaume I, creo que es necesario promover la ruptura de esta dicotomía que lleva al olvido de saberes masculinos y femeninos que considero son igual de necesarios para la construcción de una Cultura para hacer las Paces.

La relación de las mujeres con la paz no ha evitado que, incluso, sus saberes se hayan olvidado en la búsqueda de la paz. Por esta razón, afirmo que se hace necesaria la reconstrucción de sus habilidades para la propulsión de la convivencia armónica, así como el incremento de su participación en los procesos de paz. Pienso que esto debería ser así, pero no porque las mujeres tengan más destrezas que los hombres para el establecimiento del valor de la paz, sino porque como seres humanos todos y todas tenemos habilidades para hacernos las paces y las guerras, aunque sean las primeras de estas capacidades las que 
me interesan destacar en este trabajo. Se requiere, entonces, la combinación de las destrezas masculinas y femeninas si se quiere alcanzar un mayor éxito en la puesta en práctica de alternativas que busquen la creación de la paz y, también, la transformación de los conflictos por medios pacíficos ${ }^{9}$.

Las siguientes páginas sintetizan las aportaciones de las mujeres a la regulación positiva de las situaciones conflictivas. Como se ha mencionado en la introducción, esto supone uno de los marcos de acción que favorecerá la reconstrucción de la identidad de la mujer que se está proponiendo.

\subsection{La metodología de la transformación pacífica de los conflictos}

Antes de analizar en qué medida podría favorecer la práctica exitosa de la transformación pacífica de los conflictos el hecho de recordar los saberes de las mujeres y su participación, me parece interesante mencionar algunas cuestiones generales que nos ayudarán a situar la metodología de la transformación pacífica en el marco de los Estudios de los Conflictos, así como sus características principales.

Tres son las terminologías que se han venido usando para referirse a los Estudios de los Conflictos. En el siguiente esquema se muestra cada una de ellas, y se resalta el momento en el que aparecen, así como algunos de sus rasgos ${ }^{10}$ :

\begin{tabular}{|c|c|}
\hline \multicolumn{2}{|c|}{ FUNDACIONES } \\
\hline Terminología & Hechos \\
\hline $\begin{array}{l}\text { Resolución de Conflictos } \\
\text { Desde la década de } 1950 \text { a la de } 1960\end{array}$ & $\begin{array}{c}\text { Se crea el Peace Research Laboratory. } \\
\text { Aparece el Journal of Conflict Resolution. } \\
\text { Surgen las teorías de Kenneth Boulding, Johan } \\
\text { Galtung y John Burton. }\end{array}$ \\
\hline \multicolumn{2}{|c|}{ CONSTRUCCIÓN } \\
\hline Terminología & Hechos \\
\hline $\begin{array}{l}\text { Gestión de Conflictos } \\
\text { Desde la década de } 1970 \text { a la de } 1980\end{array}$ & $\begin{array}{c}\text { Tienen lugar trabajos importantes en la } \\
\text { Universidad de Harvard. } \\
\text { Surgen los estudios de Adam Curle y Elise } \\
\text { Boulding. }\end{array}$ \\
\hline \multicolumn{2}{|c|}{ RECONSTRUCCIÓN } \\
\hline Terminología & Hechos \\
\hline $\begin{array}{l}\text { Transformación de Conflictos } \\
\text { A partir de las década de } 1990\end{array}$ & $\begin{array}{l}\text { Se estructuran nuevas metodologías de } \\
\text { regulación de conflictos basadas en medios } \\
\text { pacíficos que enfatizan la cooperación, la } \\
\text { percepción, el diálogo, el reconocimiento y el } \\
\text { empoderamiento. }\end{array}$ \\
\hline
\end{tabular}

9. MARTÍNEZ GUZMÁN, Vicent: Filosofía para hacer las paces, Barcelona, Icaria, 2001.

10. Mal, Hugh; Ramsbotham, Oliver; Woodhouse, Tom: Contemporary Conflict Resolution. The prevention, management and transformation of deadly conflicts, Malden, Polity Press, 1999. 
La resolución de conflictos ${ }^{11}$ enfatiza la interpretación negativa de las situaciones conflictivas debido a que hace hincapié en la búsqueda de soluciones, y en las consecuencias destructivas que de ellas se derivan. Por lo tanto, conserva la concepción tradicional de los conflictos como fenómenos sociales que se deben evitar a toda costa. Las críticas a la resolución surgieron cuando se empezó a cuestionar si sería posible resolver todas las situaciones conflictivas e, incluso, si las resoluciones forzosas serían deseables. Sin embargo, es importante mencionar que, a pesar de estas denuncias, el concepto de «resolución" ha sido el término más aceptado, y de este modo se observa en los distintos trabajos elaborados sobre el tema.

La gestión de conflictos introduce una percepción más positiva de las situaciones conflictivas cuando supone que todo conflicto puede ser manejado mediante leyes, dinámicas o modelos. En este caso, se considera que los conflictos son fenómenos naturales, al igual que el resto de los elementos de la naturaleza. No obstante, esta metodología fue, también, criticada por aquellos quienes piensan que los conflictos son parte de las acciones humanos, y como tales, no pueden ser manejados de la misma forma que las cosas del mundo físico. Además, los críticos observan que la vinculación de la gestión de conflictos con el mundo empresarial acentúa sus aspectos teóricos y prácticos de una manera desmesurada.

En último lugar, la transformación pacífica de los conflictos será la terminología y metodología que se defenderá en este trabajo porque se entiende como la más próxima a la construcción de una Cultura de la Paz. Ello se debe a que permite percibir los conflictos como situaciones de aprendizaje, gracias al uso de medios pacíficos que hacen posible su regulación positiva, tales como la comunicación, el empoderamiento y el reconocimiento. Schnitman ${ }^{12}$ la define como "prácticas emergentes» en las que las personas «reconstruyen sus cursos de acción, sus relaciones y se reconstruyen a sí mismos», a partir de la superación de las tensiones en desacuerdo por medios pacíficos.

El objetivo que se quiere destacar es que la metodología de la transformación llevará a una interpretación positiva de las situaciones conflictivas debido a que utiliza medios pacíficos para su manejo constructivo, y con ello, evita el uso de la violencia y las consecuencias destructivas que de ella se derivan. Cabe señalar que la percepción que se tiene de los conflictos variará en función de los medios que son empleados para su propia regulación.

Algunas de las características generales de la transformación son las que siguen:

1. El objetivo de la transformación pacífica de los conflictos es la reconciliación de las partes tras la superación del conflicto. Para ello, se recuerdan las tensiones que lo causaron con el fin de convertirlo en una situación de aprendizaje, que

11. IEDERACH, John Paul: Preparing for Peace. Conflict Transformation Across Cultures, New York, Syracuse University Press, 1995.

12. SChnitman, Dora Fried: Nuevos paradigmas en la resolución de conflictos. Perspectivas y prácticas, Barcelona, Ediciones Granica, 2000, p. 22. 
permite el fortalecimiento de las relaciones entre las personas afectadas. Fisas ${ }^{13}$ afirma que "transformar el conflicto significa actuar sobre el mismo para que los aspectos creativos del mismo sean los dominantes».

2. Su método es el diálogo y la comunicación. Schnitman ${ }^{14}$ piensa que los procesos de transformación pacífica de las situaciones conflictivas "[...]se asientan en la comunicación y las prácticas discursivas y simbólicas que promueven diálogos transformativos».

3. Su práctica supone un gran esfuerzo y dedicación por parte de las personas afectadas ${ }^{15}$.

4. Las alternativas de regulación positiva que sugiere la transformación pacífica de los conflictos recuerdan todas las necesidades e intereses de las partes involucradas, porque lo que se pretende es beneficiar de igual manera, y en la medida de lo posible, a quienes se encuentran implicados.

5. Los cambios alcanzados con la transformación pacífica de los conflictos han de ser perdurables y sostenibles en el tiempo ${ }^{16}$.

6. La visión positiva de los conflictos que se constituye con su transformación pacífica no elimina de forma absoluta el sufrimiento de las personas implicadas. Aunque los motivos de este sufrir son muy diferentes a los causados con el uso de la violencia como medio de regulación, es cierto que se puede seguir hablando de sufrimiento en el transcurso de esta vivencia positiva.

7. En resumen, se cree necesario incidir en las siguientes estrategias si se quiere lograr una práctica exitosa de los presupuestos de la transformación pacífica de los conflictos: crear una atmósfera óptima para el diálogo y la comunicación; describir la naturaleza del conflicto desde todas sus perspectivas; comprender las razones por las que las partes contrarias tienen una actitud determinada; analizar todas las causas que han llevado al conflicto, las necesidades de cada parte y cuáles no están siendo satisfechas; buscar decisiones basadas en los criterios de igualdad y simetría; y desarrollar alternativas que permitan la satisfacción de las necesidades de ambas partes, y el inicio de una acción diseñada para encontrar metas comunes ${ }^{17}$. Para la consecución de estas dinámicas se requiere, tal y como diría Porro ${ }^{18}$, "Hablar hasta entenderse», porque se considera que la comunicación y el discurso juegan un papel central en la práctica de la transformación pacífica de los conflictos.

13. HSAS, Vicenç: Cultura de paz y gestión de conflictos, Barcelona, Icaria, 2002, p. 233.

14. SCHNitMAn, Dora Fried: Op. cit, p. 25.

15. IEDERACH, John Paul: Preparing for Peace. Conflict Transformation Across Cultures..., Op. cit.

16. Iederach, John Paul: Building Peace. Sustainable Reconciliation in Divided Societies, Washington, United States Institute of Peace, 1998.

17. KotTler, Jeffrey A.: Beyond Blame: a new way of resolving conflicts in relationships, San Francisco, Jossey-Bass Publications, 1994, p. 8.

18. PORRO, Bárbara: La Resolución de Conflictos en el Aula, Buenos Aires, Paidós, 2000. 


\subsection{Repensar la identidad de la mujer y su papel en la transformación pacífica de los conflictos}

En la línea de las ideas que he venido mencionando en las páginas anteriores, me propongo repensar la identidad de las mujeres con el fin de reconstruir sus aportaciones a la transformación pacífica de los conflictos. Tener en cuenta las posibilidades de participación de las mujeres en la construcción de la paz nos permitirá reconstruir su identidad desde una perspectiva más activa, dinámica y comprometida con las problemáticas actuales. En este sentido, afirmo que las mujeres pueden promover contribuciones que favorecerán, en gran medida, la práctica exitosa de las características principales de la regulación pacífica de los conflictos, debido a la forma en la que, tradicionalmente, han sido educadas y socializadas, y a los límites que se han impuesto a su presencia en los ámbitos públicos y sociales. Cabe recordar que esto no supone que la transformación pacífica de los conflictos dependa, exclusivamente, de los saberes de las mujeres, sino que su práctica puede verse beneficiada si se tienen en cuenta tanto sus experiencias y conocimientos como los de los hombres.

Los rasgos de la transformación pacífica de los conflictos que considero se verán favorecidos con la participación y colaboración de las mujeres son los siguientes:

1. El desarrollo del empoderamiento. Este concepto se desarrolla en el marco de las teorías feministas que proponen la reconstrucción de las habilidades de las mujeres a fin de afrontar las situaciones de subordinación en las que viven. De esta forma, podría servir como una alternativa para la regulación de la violencia de género que, en muchas ocasiones, hemos visto que se deriva como consecuencia del miedo a lo diferente ${ }^{19}$.

Por lo tanto, el empoderamiento facilitaría a las mujeres la recuperación de sus habilidades y capacidades para la regulación positiva de los conflictos, al mismo tiempo que sus aportaciones favorecerían la práctica exitosa de la transformación pacífica de los conflictos. En ocasiones, también se podría requerir la ayuda de una tercera persona que haría las funciones de "facilitadora» en la búsqueda de los acuerdos comunicativos, aunque siempre deberían ser las partes implicadas las que escogiesen las soluciones más favorables para los conflictos que las afectan.

Encontramos otros conceptos que tienen el mismo significado que el término de empoderamiento. Por ejemplo, en la versión española del libro de Bush y Folge ${ }^{20}$ se habla de revalorización, Lederach ${ }^{21}$ utiliza el término de capacitación, y el Programa de Naciones Unidas al Desarrollo el de potenciación.

En resumen, la habilidad del empoderamiento necesaria para la transformación pacífica de los conflictos se ve favorecida con la participación y las apor-

19. REARDON, Betty: Op. cit.

20. BuSH, Robert A. Baruch; FOLGER, Joseph P.: La promesa de la mediación. Cómo afrontar el conflicto mediante la revalorización y el reconocimiento, Barcelona, Granica, 1996.

21. IEDERACH, John Paul: Preparing for Peace. Conflict Transformation Across Cultures..., Op. cit. 
taciones de las mujeres, quienes además son capaces de afrontar sus miedos con mayor éxito. Como se ha mencionado al citar a Reardon ${ }^{22}$, proponemos afrontar nuestro miedo al diferente, nuestra vulnerabilidad y fragilidad humana por medios pacíficos con el fin de posibilitar la construcción de la cultura de la paz. En este caso, también encontramos actitudes que podríamos reconocer y aprender de las mujeres.

"Según lo definen Bush y Folger, empowerment implica ayudar a cada parte a que desarrolle "un mayor sentimiento de autovaloración, seguridad, autodeterminación, y autonomía' (87). Esto se logra mediante la habilidad del mediador de manifestar respeto y escuchar atentamente a cada disputante; y en mayor medida, aun, brindando oportunidades para la recuperación del poder, manteniendo durante todo el proceso el poder de decisión en manos de los disputantes» ${ }^{23}$.

2. El devenir de la comunicación. Las habilidades comunicativas de las mujeres facilitarán el desarrollo del modelo de comunicación que considero adecuado para la transformación pacífica de los conflictos. A partir de la Teoría de los Actos de Habla de Austin ${ }^{24}$, afirmo que todo "decir es un hacer", y que por lo tanto, deberíamos ser muy cuidadosos con las formas en las que nos decimos o no nos decimos las cosas. Esto es así porque una de las principales causas del surgimiento de los conflictos es la falta de comunicación, el mal uso que hacemos de nuestras palabras, gestos y silencios, y la mala interpretación de los mensajes que nos transmitimos.

El objetivo es promover un modelo de diálogo y comunicación acorde a la regulación positiva de los conflictos que transforme estas causas por medios pacíficos, y que permita alcanzar el entendimiento lingüístico entre las partes, así como acuerdos comunicativos que sean propicios a todas ellas. Todo ello con el fin de satisfacer los intereses y necesidades de las diferentes personas afectadas de forma igualitaria, y en la medida que sea posible, por medio de respuestas cooperativas, del entendimiento de las percepciones ajenas y del uso de formas de poder no autoritarias ni subordinantes.

El modelo de diálogo que considero necesario tendrá en cuenta los presupuestos de la Ética del Discurso, en general, y de las Teorías de los Conflictos, en particular, y se verá favorecido con la participación y las contribuciones de las mujeres.

22. REARDON, Betty: Op. cit.

23. SHAILOR, Jonathan G.: «Desarrollo de un Enfoque Transformador para la Mediación: Consideraciones Teóricas y Prácticas», en Dora Fried Schnitman (ed.): Nuevos paradigmas en la resolución de Conflictos. Perspectivas y prácticas, Barcelona, Granica, 2000, p. 186.

24. Austin, John L.: Palabras y acciones. Cómo hacer cosas con palabras, Buenos Aires, Paidós, 1971. 


\section{Características del Diálogo para la Transformación Pacífica de los Conflictos}

1. Recuerda la libertad y la igualdad de las partes durante el proceso de la comunicación.

2. Pone el énfasis en las tres pretensiones de validez del habla que se señalan desde la Ética del Discurso: la verdad de los contenidos, la veracidad de la intención y la corrección del componente realizativo*.

3. Hace hincapié en la participación cooperativa de las personas implicadas.

4. Supone que las partes admiten la responsabilidad de las cosas que estamos diciendo y de las consecuencias que de ellas se derivan.

5. Ofrece importancia a la expresión personal. Si queremos que el diálogo sea exitoso, cada persona debe decir todo lo que piensa y siente**.

6. Concede valor y respeto a las otras partes afectadas, de tal forma que debemos escuchar a las otras personas con la finalidad de reconocerlas. Las posibilidades de transformación de una situación conflictiva aumentan si la «reafirmación» permite la consecución del acuerdo.

7. Requiere acciones coordinadas. Es decir, este tipo de diálogo prospera gracias a la coordinación mutua que tiene lugar cuando las acciones de una persona en la conversación sirven para convalidar los de la otra.

8. Potencia la autoreflexividad. Con esta actitud se hace posible el surgir de nuevas ideas durante la conversación y el diálogo.

9. Pretende facilitar la creación colaborativa de nuevas realidades. Necesita de otros momentos «imaginarios» en los que los participantes tratan de localizar otras visiones de la realidad.

\section{Técnicas para la puesta en marcha del diálogo transformador según Bodine, Crawford} y Schrumpf***

1. Escuchar activamente no sólo para entender lo que se dice, sino, también, para comprender las percepciones, emociones y el contexto del que se habla, así como para mostrar que uno ha entendido lo que se ha dicho.

2. Hablar con el propósito de hacerse entender.

3. Hablar sobre uno mismo y no sobre el otro.

4. Hablar con una finalidad bien establecida.

5. Hacer ajustes que recuerden las diferencias de personalidad, sexo y cultura.

* CoRtina, Adela: Crítica y utopía a la escuela de Franckfort, Madrid, Ediciones Pedagógicas, 1994.

** SChnitman, Dora Fried: Nuevos paradigmas en la resolución de Conflictos. Perspectivas y prácticas, Barcelona, Granica, 2000. Las características 5, 6, 7, 8 y 9 se incluyen siguiendo las investigaciones de Schnitman en este libro.

*** GRARD, Kathryn; KoCH, Susan J.: Resolución de conflictos en las escuelas. Manual para educadores, Barcelona, Granica, 1997.

Siguiendo las ideas mencionadas anteriormente, los silencios y la comunicación no verbal juegan un papel importante en las formas en las que nos decimos las cosas, ya que, también, podemos hacernos muchas cosas con todo aquello que no nos decimos. Así, en este modelo de comunicación que vengo elaborando es importante recordar los dos principios para una Axiomática de una nueva Filología propuestos por Ortega y Gasset, según los que: a) Todo decir es deficiente porque dice menos de lo que quiere. b) Todo decir es exuberante porque da a entender más de lo que se propone. Los seres humanos no podemos decirlo todo, y por ello, la actividad del decir es una faena utópica «en el sentido de que nunca vamos a poder decirlo todo, decir siempre será un proceso hacia el decirlo todo que nunca alcanzaremos» ${ }^{25}$. Como consecuencia, se distinguen

25. MARTíneZ GUZMÁN, Vicent: "El silencio como interpelación: paz y conflicto», en Mary Farrell y Dora Sales (eds.): El silencio en la comunicación humana, Castellón, Seminari d'Investigació Feminista. Universitat Jaume I, 1999, p. 116. 
tres tipos de silencios: 1) El silencio de lo inefado que es aquello que se calla por sabido. 2) El silencio de lo inefable que es el silencio de aquello que no podemos decir. De este segundo tipo se desprenden dos formas más que son 2.1) Aquello que es inefable por ser complicado, sublime o divino, y 2.2) Los inefables triviales que muestran el silencio como condición de posibilidad de lenguaje. 3) El silencio gestual donde aparecen los gestos callados que acompañan el lenguaje o que dicen cosas por sí mismos.

"Soy violento y ofendo con mis palabras y mis silencios cuando suponen una exclusión del otro, una falta de reconocimiento del otro que aparece sólidamente ligado a mí en todas mis acciones como ser humano. Soy capaz de entender que soy violento, que mi silencio puede ser violento, porque altero lo que es originario de las relaciones humanas, a saber, la intersubjetividad solidaria ${ }^{26}$.

3. El transcurso de la responsabilidad. La transformación de los conflictos por medios pacíficos requiere actitudes basadas en la responsabilidad. Es decir, supone que debemos responsabilizarnos de las cosas que nos hacemos, decimos y callamos, y de las consecuencias que de ellas se derivan. Por esta razón, considero que el papel que las mujeres han tenido en el marco de la vida privada, también, puede favorecer el devenir de la responsabilidad en el ámbito de la regulación positiva de los conflictos.

En este sentido, las contribuciones de las mujeres podrían facilitar el desarrollo de nuestra responsabilidad desde tres puntos de vista, que se configuran siguiendo la Fenomenología Lingüística de Strawson ${ }^{27}$ : 1) El de cómo me siento por lo que me hacen a mí. Son diferentes las formas en las que nos podemos sentir cuando aquellos quienes nos rodean deciden hacernos alguna cosa. En este sentido, en función de esas acciones sentiremos gratitud, alegría, rencor u odio. Propongo una transformación de los «sentimientos negativos» (que llevan a la violencia y a la ruptura de las relaciones) en beneficio de "sentimientos positivos» (que favorecen la práctica de la transformación pacífica de los conflictos). 2) El de cómo me siento por lo que una segunda persona hace a una tercera persona. Se deberían tener en cuenta, también, aquellas acciones que no nos afectan de forma directa, pero que suceden en el mundo, y por lo tanto, podrían perjudicarnos en un futuro inmediato. Cabe decir que esta responsabilidad es importante no sólo porque dichas acciones podrían llegar a afectarnos, sino porque se quiere poner el énfasis en la capacidad de indignación que como seres humanos tenemos, y que, por lo tanto, tendríamos que aprovechar con el fin de promover actitudes favorables a la paz. 3) El de cómo me siento por lo que yo hago. Asimismo, son diversos los sentimientos que surgen ante las acciones que realizamos en función de las personas a las que las dirigimos, y de los intereses que las promueven. $\mathrm{Al}$ igual que se ha dicho anteriormente, propongo los «sentimientos positivos»

26. Ibíd, p. 112.

27. StraWSON, Peter: "Freedom and Resentment", en Peter Strawson (ed.): Freedom and Resentment and Other Essays, London, Methuen, 1974, pp. 1-25. 
como aquellos que deberían ser resaltados en el transcurso de las relaciones humanas.

En mi interpretación, considero que estas tres actitudes se verían facilitadas con la participación activa y comprometida de las mujeres en la transformación pacífica de los conflictos.

«Siempre podemos pedirnos responsabilidad por lo que decimos que es la guerra, los discursos que hacemos, las cosas que hacemos con las palabras, y siempre está la posibilidad de preguntarnos si podemos hacérnoslas de otra manera. Lo que tiene interés para la filosofía para la paz es qué decirnos de la guerra, qué metáforas usamos, cómo la hemos aprendido, qué hacemos con las palabras, a qué nos comprometemos, qué dejamos en los márgenes, a quién excluimos, etc» ${ }^{28}$.

4. El desarrollo del reconocimiento. La contribución de las mujeres, también, facilitaría el devenir del reconocimiento en la transformación pacífica de los conflictos. Siguiendo a Honneth ${ }^{29}$ resaltamos tres tipos de reconocimiento que deberíamos tener en cuenta para el establecimiento del valor de la paz:

4.1. El reconocimiento de la integridad física de las personas, que implica la capacidad de reconocernos físicamente con nuestras particularidades y especificidades propias. En este caso, podríamos hablar de un reconocimiento del cuerpo de la mujer basado en actitudes no violentas, que no derivarían del miedo a lo diferente ${ }^{30}$. Esto es posible cuando se hace uso del sentimiento del amor como aquel que representa el primer estadio del reconocimiento recíproco porque favorece que cada individuo confirme su propia naturaleza como un ser que es, al mismo tiempo, capaz de amar y de ser amado. Cabe decir que este tipo de relación va unida a la existencia corporal del otro y, por esta razón, se concibe como un «ser en sí mismo en el otro». Asimismo, este "ser en el otro» implica que todas las personas estamos necesitadas del reconocimiento por aquella otra persona con quien nos identificamos. Es decir, todas las relaciones amorosas se alimentan de aquella primera vivencia originaria que tiene lugar entre una madre y su hijo ${ }^{31}$.

El amor representa, por lo tanto, el primer tipo de reconocimiento que favorece el devenir de la autoconfianza, y la clase de menosprecio que impide llevarlo a término es aquel que se provoca cuando a una persona se le impide hacer uso de su cuerpo libremente. Un ejemplo de este tipo de menosprecio sería la violación. Es decir, el momento en el que una mujer es tomada a la fuerza y se le retira la capacidad de decidir libremente qué hacer con su propio cuerpo.

4.2. El reconocimiento de las personas como individuos que forman parte de una comunidad, y como tales son poseedores de una serie de derechos. Se refiere a las relaciones de derecho porque propone el reconocernos todos y todas como

28. Martínez GuZmán, Vicent: «Deconstruir la Guerra, Reconstruir la Paz», en Vicente J. Benet y Vicente Sánchez Biosca (eds.): Decir, Contar, Pensar la Guerra, Valencia, Generalitat Valenciana, Ediciones Cimal Arte Internacional, 2001, p. 166

29. HDNNETH, Axel: La lucha por el reconocimiento, Barcelona, Crítica, 1997.

30. REARDON, Betty: Op. cit.

31. HDNNETH, Axel: Op. cit. 
personas de derecho que tenemos derecho a la libertad, participación política, etc ${ }^{32}$

Esta clase de reconocimiento favorece el surgimiento del autorespeto, y la clase de menosprecio que impide su desarrollo es aquel que se produce cuando un sujeto es excluido de determinados derechos dentro de una sociedad. Un ejemplo de este tipo de menosprecio sobre el que podríamos reflexionar sería la subordinación que han vivido las mujeres cuando se han visto relegadas a la esfera de lo privado, sin casi participación en la esfera de lo público.

4.3. El reconocimiento a las diferentes formas de vida en el sentido de solidaridad. Es decir, se propone, también, el entendimiento de las distintas maneras de vida con el fin de favorecer la transformación pacífica de los conflictos. En este caso, las formas en las que, tradicionalmente, se ha educado a las mujeres pueden facilitar la comprensión y el desarrollo de la solidaridad ${ }^{33}$.

Este tipo de reconocimiento favorece el surgimiento de la autoestima, y la forma de menosprecio que impide su avance es aquel según el que se niega la comprensión de las percepciones e intereses que nos son ajenos. Por ejemplo, esta clase de menosprecio tiene lugar cuando hay una falta de entendimiento de otras tradiciones culturales.

En resumen, la participación de las mujeres en la transformación pacífica de los conflictos facilitará la búsqueda de alternativas favorables a la convivencia armónica, así como la construcción de una cultura para hacer las paces. Esto nos permitirá tener una imagen de la mujer más activa, participativa y comprometida, así como reconstruir su identidad en el marco de las sociedades actuales.

\section{CONCLUSIÓN}

El objetivo de este trabajo ha sido mostrar de qué manera la transformación pacífica de los conflictos puede verse favorecida si se tienen en cuenta las aportaciones y la participación de las mujeres. Así, se ha considerado que la regulación positiva de los conflictos puede ser un marco de acción de las mujeres que facilitará la construcción y el establecimiento de una Cultura para hacer las Paces. Sin embargo, no se ha querido señalar que la metodología de la transformación debería ser, exclusivamente, femenina, sino que su práctica debería tener en cuenta tanto los conocimientos y las experiencias de las mujeres como las de los hombres.

La tesis general que se ha manejado es que todos y todas tenemos habilidades para hacernos las paces y las guerras. No obstante, se han destacado las primeras de estas capacidades como aquellas que nos llevarán a la propulsión de una convivencia armónica. De este modo, si todos y todas tenemos estas habilidades no deberíamos excluir el papel que las mujeres pueden desempeñar en el marco de la transformación pacífica de los conflictos. El valor de la paz supone un esfuerzo conjunto, así como una combinación de los saberes femeninos y masculinos.

32. Ibíd.

33. Ibíd. 
A partir de esta hipótesis de trabajo y desde una perspectiva fenomenológica, se ha hecho un recorrido por las influencias que ha tenido el cuerpo de la mujer en las formas en las que, tradicionalmente, ha sido percibido. En este caso, se ha observado que el miedo a lo diferente ha sido, en muchas ocasiones, la causa de la violencia de género. Por esta razón, se ha propuesto afrontar este miedo, ya no sólo a lo diferente, sino, también, a la vulnerabilidad y fragilidad humana por medios pacíficos.

Finalmente, se han revisado cuatro rasgos de la transformación pacífica de los conflictos que se verían favorecidos con las contribuciones de las mujeres. De este modo, se ha destacado el empoderamiento, la comunicación, la responsabilidad y el reconocimiento. Recordar la participación de las mujeres y sus aportaciones ayudarian no sólo a la práctica exitosa de la transformación, sino, también al establecimiento de la cultura para hacer las paces. Asimismo, todo ello nos ha permitido reconstruir la identidad de las mujeres de una forma más dinámica, activa y comprometida con el valor de la paz y con las problemáticas de la sociedad actual. 